\title{
A STUDY TO REVIEW THE APPROPRIATENESS OF DRUG DOSAGE IN RENALLY IMPAIRED PATIENT BY IDENTIFYING, ANALYSING, AND ADJUSTING DOSAGES IN A TERTIARY CARE TEACHING HOSPITAL
}

\author{
RATHOD MRUDANGSINH M, ANCY ACHANKUNJU, ROSE MARIA JOHN, GLORIA K SAM*
}

Department of Pharmacy Practice, Bapuji Pharmacy College, Davanagere, Karnataka, India. Email: gloria_sam52@yahoo.com

Received: 02 January 2017, Revised and Accepted: 27 January 2017

ABSTRACT

Objective: The objective of this study is to identify and analyze drug dosing in renally impaired patient in a tertiary care teaching hospital and to categorize patients on the basis of gender, age, glomerular filtration rate (GFR), and anemia.

Methods: A prospective, interventional study was conducted on the drug dosage in renally impaired patient by identifying, analyzing, and adjusting. Ethical clearance was obtained from the Institutional Ethical Committee. The study was conducted on 103 patients with renal impairment for 6 months. During ward rounds, cases were collected, and collected data were assessed and analyzed using Micromedex, Lexicomp. For the possible interventions, the dosage errors were reported along with the evidence.

Results: Out of the 103 patients, 73 were males, and 30 were females, and majority of patients belonged to the age group between 41 and 65 years. About $56.31 \%$ and $43.69 \%$ of patients were in the Category B and Category C of GFR, respectively. A total of 244 comorbidities were present in 103 patients with hypertension and Type 2 diabetes mellitus the foremost. In total, 983 drugs were prescribed to the total patient with an average of 9.54 drugs per patient, 175 drugs required dosage adjustment.

Conclusion: The present study effectively reports all the interventions to the physicians along with the supporting data. Continuous medical education to the physicians together with pharmacists may reduce the chances of incongruity in drugs given to the renally impaired patients.

Keywords: Renal impairment, Modification of diet in renal disease, Creatinine clearance, Dosage adjustment.

(c) 2017 The Authors. Published by Innovare Academic Sciences Pvt Ltd. This is an open access article under the CC BY license (http://creativecommons. org/licenses/by/4. 0/) DOI: http://dx.doi.org/10.22159/ajpcr.2017.v10i4.16886

\section{INTRODUCTION}

Renal failure is a condition where the kidneys lose their normal functionality. It is characterized by the reduction in the excretory and regulatory functions of the kidney. It usually occurs at the terminal stages of the disease process. Kidney failure - when one or both kidneys are not able to perform their usual functions. The main function of the kidney is to remove waste from the body and to balance the water and electrolyte content of the blood by filtering the salt and water in the blood. The waste and water excreted by the kidneys combine to form urine [1].

Treatment of renal failure may include medical management, dialysis, lifestyle changes, organ transplants, and immune suppressive therapy. Lack of adherence to prescribed renal failure treatments is a public health issue, as it is a major contributor to poor outcome [2].

Types of renal failure

Acute renal failure

The kidneys abruptly stop working entirely or almost entirely but may eventually recover to nearly normal function.

\section{Chronic renal failure}

An irreversible and progressive loss of renal functions, resulting in a variety of clinical and laboratory changes due to reduced renal excretory, endocrine, and regulatory functions [1].

Unpleasant effects of renal impairment

Impairment or degeneration of kidney function affects the pharmacokinetics of drugs. Some of the more common causes of kidney failure include disease, injury, and drug intoxication. Acute diseases or trauma to the kidney can cause uremia, in which glomerular filtration is impaired or reduced, leading to accumulation of excessive fluid, and nitrogenous products in the body. Uremia generally reduces glomerular filtration and/or active secretion, which leads to a decrease in renal drug excretion resulting in a longer elimination half-life of the administered drug [3].

Decreased drug clearance results in higher drug concentrations and hence greater drug effects. To avoid harm when drug clearance is significantly decreased, the dose of renally cleared drugs should generally be reduced in patients with renal disease [4].

\section{Assessment of kidney function \\ - $\quad$ Serum creatinine}

- $\quad$ Creatinine clearance [5].

\section{Dosage adjustment in renal impairment}

Dosage regimen adjustment is mainly considered in renal diseases, when the drug is mainly (at least $70 \%$ of the dose) excreted by the kidney either unchanged or as an active metabolite or the therapeutic window of the drug or the metabolite is narrow. Dosage regimen adjustment options involve (i) reduction of the dose level, (ii) extension of the dosing interval, (iii) administration of a loading dose, and/or (iv) therapeutic drug monitoring. The proper dosing of medications for patients with renal impairment can maximize therapeutic efficacy and minimize toxicity. Proper dosing can also have an economic impact on the health system [6].

\section{Objectives}

To identify and analyze drug dosing in renally impaired patient in a tertiary care teaching hospital and to categorize patients on the basis of gender, age, glomerular filtration rate (GFR), and anemia. 


\section{METHODS}

A prospective, interventional study was conducted for 6 months in the Nephrology Department, Emergency Unit, Intensive Care Unit, general wards and dialysis unit of a tertiary care teaching hospital. All renally impaired patients above the age of 18 years of either sex with serum creatinine above $1.4 \mathrm{mg} / \mathrm{dl}$ or GFR below $50 \mathrm{ml} / \mathrm{min} / 1.73 \mathrm{~m}^{2}$ were included in the study, whereas patients with chronic kidney disease (CKD) Stage 1 and 2 along with pregnant women and comatose patients were excluded from the study.

\section{Ethical issues}

The ethical clearance for the study was obtained from Institutional Ethical Committee of Bapuji Pharmacy College, Davangere.

\section{Study procedure}

The ward rounds were attended every day along with a nephrologist and patient details from patient profile form was collected to specially designed data collection form. The collected data was assessed and analyzed to identify dosing errors in medication chart using Martindale Complete Drug Reference, Micromedex, Lexicomp, British National Formulary and Research articles and Journals. For possible interventions, the dosage errors were reported to the nephrologist and other consulting physicians along with supporting data. The collected data were analyzed by applying suitable statistical method.

\section{RESULTS}

A total of 103 patients prescriptions were collected, out of which 73 (70.87\%) were males and $30(29.13 \%)$ were females. Out of 103 patients analyzed, majority of patients were in the age group of 41-65 years $(n=62,60.19 \%)$, followed by $>65$ years $(n=21,20.39 \%)$, and $18-40$ years $(n=20,19.42 \%)$. Among the total 103 patients, 58 patients $(56.31 \%)$ belong to the Category B, followed by 45 patients (43.69\%) in the Category C while analyzing the GFR. Out of 103 patients, 54 patients $(52.43 \%)$ have moderate anemia followed by 37 patients $(35.92 \%)$ with mild anemia and 12 patients $(11.65 \%)$ with severe anemia. Totally, 40 patients (38.83\%) among the 103 patients were found with two comorbidities, followed by 27 patients $(26.21 \%)$ with three comorbidities, 22 patients (21.36\%) with one comorbidity, 10 patients $(9.70 \%)$ with four comorbidities, 3 patients $(2.93 \%)$ with five comorbidities, and only 1 patient $(0.97 \%)$ with six comorbidities. Average number of comorbidities per patient was 2.37. A total of 244 comorbidities were present in 103 patients, with hypertension (HTN) (27.87\%) and Type 2 diabetes mellitus (DM) (19.67\%) the foremost, followed by cardiovascular disorders, respiratory tract infections, urinary disorders, and central nervous system disorders.

In total, 983 drugs were prescribed to the entire 103 study population with an average of 9.54 drugs per patient. Minimum number of drugs prescribed to the patient was 5 and maximum were 17 . Among the 103 study population, 16 patients received 10 drugs per prescription. Out of $983(100 \%)$ drugs prescribed to patients, 175 (17.80\%) drugs required dosage adjustment (Table 1 ).

Out of 175 drugs requiring dosage adjustment, only 92 drugs were prescribed in adjusted dose. From the total 103 renally impaired patients, 51 patients $(49.51 \%)$ required interventions. Antimicrobials (32\%) are the most frequently prescribed class of drug which require dosage adjustment followed by antihypertensives (22.86\%), antiplatelet (6.29\%), opioids (5.14\%), antiepileptics (4.57\%), antiemetics (4\%), $\mathrm{H}_{2}$ receptor antagonist $(2.29 \%)$, genitourinary agents $(2.29 \%)$, oral hypoglycemic agents (1.71\%), calcium supplements (1.14\%), nootropic agents $(1.14 \%)$, antihistaminics $(0.57 \%)$, antipsychotics $(0.57 \%)$, xanthine oxidase inhibitor $(0.57 \%)$, and cardiovascular agents $(0.57 \%)$ (Table 2).

From the 56 antimicrobials requiring adjustments, 31 were not adjusted properly. Among commonly prescribed antimicrobials, the main class which was not modified as per the creatinine clearance was fluoroquinolones followed by nitro compounds (Table 3).

Among the 40 antihypertensive drugs requiring adjustments, 13 drugs were not adjusted, with diuretics being the foremost (Table 4).

\section{DISCUSSION}

The study was conducted for a period of 6 months and data of 103 patients, who satisfied the inclusion criteria, were analyzed in a prospective manner. The degree of renal impairment was assessed

Table 1: Determination of requirement of dosage adjustment in renally impaired patients

\begin{tabular}{lll}
\hline $\begin{array}{l}\text { Total number of } \\
\text { drugs }\end{array}$ & $\begin{array}{l}\text { Number of drugs } \\
\text { requiring dosage } \\
\text { adjustment }\end{array}$ & $\begin{array}{l}\text { Percentage of drugs } \\
\text { requiring dosage } \\
\text { adjustment }\end{array}$ \\
\hline 983 & 175 & 17.80 \\
\hline
\end{tabular}

Table 2: Distribution of drugs requiring dosage adjustments in renally impaired patients

\begin{tabular}{lll}
\hline S.No. & Class of drugs & Number of drugs $\mathbf{n = 1 7 5}(\mathbf{0})$ \\
\hline 1 & Antimicrobials & $56(32)$ \\
2 & Antihypertensives & $40(22.86)$ \\
3 & Analgesics and antipyretics & $25(14.29)$ \\
4 & Antiplatelet & $11(6.29)$ \\
5 & Opioids & $9(5.14)$ \\
6 & Antiepileptics & $8(4.57)$ \\
7 & Antiemetics & $7(4)$ \\
8 & H receptor antagonist & $4(2.29)$ \\
9 & Genitourinary agents & $4(2.29)$ \\
10 & OHA & $3(1.71)$ \\
11 & Calcium supplements & $2(1.14)$ \\
12 & Nootropic agents & $2(1.14)$ \\
13 & Antihistaminics & $1(0.57)$ \\
14 & Antipsychotics & $1(0.57)$ \\
15 & Xanthine oxidase inhibitor & $1(0.57)$ \\
16 & Cardiovascular agents & $1(0.57)$ \\
\hline
\end{tabular}

OHA: Oral hypoglycemic agents

Table 3: Distribution of antimicrobials

\begin{tabular}{llll}
\hline S.No. & $\begin{array}{l}\text { Class of } \\
\text { antimicrobials }\end{array}$ & $\begin{array}{l}\text { Requiring } \\
\text { adjustment }\end{array}$ & Not adjusted \\
\hline 1 & Penicillins & 18 & 4 \\
2 & Fluoroquinolones & 14 & 14 \\
3 & Cephalosporins & 9 & 3 \\
4 & Nitro compounds & 8 & 7 \\
5 & Carbapenems & 2 & - \\
6 & Sulfonamides & 1 & 1 \\
7 & Aminoglycosides & 1 & 1 \\
8 & Imidazole agonists & 1 & - \\
9 & Polymyxin antibiotic & 1 & 1 \\
10 & Macrolide antibiotic & 1 & - \\
\hline
\end{tabular}

Table 4: Distribution of antihypertensives

\begin{tabular}{llll}
\hline S.No. & $\begin{array}{l}\text { Class of } \\
\text { antihypertensives }\end{array}$ & $\begin{array}{l}\text { Requiring } \\
\text { adjustments }\end{array}$ & Not adjusted \\
\hline 1 & Centrally acting drugs & 17 & 1 \\
2 & Diuretics & 13 & 5 \\
3 & Alpha-antagonists & 4 & 4 \\
4 & ACE inhibitors & 3 & 2 \\
5 & Vasodilators & 2 & - \\
6 & Beta blockers & 1 & 1 \\
\hline ACE: Angiotensin-converting-enzyme & &
\end{tabular}


by calculating creatinine clearance using modification of diet in renal disease equation as that of a study conducted by Sepideh et al. and Markota et al. $[7,8]$.

The study explores the dominancy of males over females in the development of renal impairment as similar to that of a study performed by Alahdal and Elberry and Henok et al. with a male population of 55\% on both $[9,10]$. Among the total 103 patients, 73 patients $(70.87 \%)$ were males and 30 patients (29.13\%) were females in our study.

The study enrolled all renally impaired patients above the age of 18 years, and majority of patients belong to the age group of 41-65 years $(60.19 \%)$ as that of a previous study carried out by Kidu et al. where the predominance of the age group 41-65 was found to be $32 \%$ [11].

As in the present study, moderate renal impaired patients (56.31\%) were also mostly reported in a study conducted by Sah et al. (62.41\%) [12]. Based on the creatinine clearance, patients were grouped into three categories as Category A (GFR $>50 \mathrm{ml} / \mathrm{min} / 1.73 \mathrm{~m}^{2}$ ), Category B (GFR: $10-50 \mathrm{ml} / \mathrm{min} / 1.73 \mathrm{~m}^{2}$ ), and Category C (GFR $<10 \mathrm{ml} / \mathrm{min} / 1.73 \mathrm{~m}^{2}$ ).

The study classified the patients based on their hemoglobin $(\mathrm{Hb})$ level as mild (Hb: 10-12 g/dl), moderate ( $\mathrm{Hb}: 6-10 \mathrm{~g} / \mathrm{dl}$ ) and severe $(\mathrm{Hb}<6 \mathrm{~g} / \mathrm{dl})$ since reduction in $\mathrm{Hb}$ level is a common phenomenon associated with kidney dysfunction. Most of the patients belong to the moderate category of anemia with $52.43 \%$.

The most frequent comorbidities linked with renal impairment are HTN and Type 2 DM since these two conditions can lead to the progressive damage of kidneys, which supports our study outcome where HTN was found to be $27.87 \%$, and Type 2 DM was found to be $19.67 \%$ of total comorbidities.

Of the 103 patients evaluated, 51 patients required interventions with the percentage of $49.51 \%$. Remaining patients were received with either already adjusted drugs or drugs that do not need any dose modification.

All patients included in the study were receiving a minimum of 5 drugs and a maximum of 17 drugs with an average of 9.54 drugs per patient. The total number of drugs received by the subjects was 983 .

Out of the 983 drugs prescribed, 175 drugs (17.8\%) required dosage adjustment, among which 92 drugs were already adjusted. One of the previous studies conducted by Erler et al. identifies 25\% requirement of dosage adjustment in drugs given to the CKD patients [13].

In the current study, the most inappropriately prescribed class of drugs was antimicrobials (32\%), and it coincides with the results of the studies conducted by Prajapati and Ganguly [14] (80\%) and Kidu et al. (59.2\%) [11]. Among the antimicrobials, dosage adjustments were required mostly for penicillins and fluoroquinolones. Antihypertensives were the class of prescribed drugs requiring dosage adjustment with centrally acting drug, clonidine being the foremost.

The study was concerned with the analysis of drugs prescribed to the renally impaired patients and to identify the appropriateness of dosage modification of renally excreted drugs by mainly using Micromedex and Lexicomp as leading sources.

\section{CONCLUSION}

To conclude, the current study provides insights into the need of dosage adjustments in renally impaired patients and appropriateness of drugs provided to them. Dosages of drugs given to the subjects were analyzed using Micromedex and Lexicomp as leading sources.

This study emphasizes the importance of improving the identification of patients with renal dysfunction, estimating their $\mathrm{CrCl}$ and implementing appropriate dose adjustments according to these estimates. This study improves patient-specific outcomes such as updated information to assist dosage guidelines, access to individuals trained in pharmacokinetics, implementation of pharmacokinetic dose consultation services in departments of clinical pharmacy, a continuous review of medication chart by the clinical pharmacist can reduce the frequency of inappropriateness in the drugs provided to the really impaired patients. The present study effectively reports all the interventions to the physicians along with the supporting data. Continuous medical education to the physicians together with clinical pharmacists can reduce the chances of inappropriateness in drugs given to the renally impaired patients.

\section{REFERENCE}

1. Modi CM, Modi FD, Mody SK, Patel HB. Pharmacokinetic of renal failure therapy. IJPRBS 2012;1(3):120-32.

2. Venkateswararao S, Asha SS, Kshama I, Rama P. Evaluation and pharmacist's intervention for improving adherence among renal failure patients. Int J Pharm Pharm Sci 2015;7(3):82-5

3. Leon S, Susanna W, Andrew B. Dosage adjustment in renal and hepatic disease. Applied Biopharmaceutics and Pharmacokinetics. $5^{\text {th }}$ ed. Ch. 21. USA: McGraw Hill Professional; 2004.

4. Doogue MP, Polasek TM. Drug dosing in renal disease. Clin Biochem Rev 2011;32(2):69-73.

5. Randall F, Lisa L. Prescribing in renal disease. Aust Prescr 2007;30(1):17-20

6. Hassan Y, Al-Ramahi R, Abd Aziz N, Ghazali R. Drug use and dosing in chronic kidney disease. Ann Acad Med Singapore 2009;38(12):1095-103.

7. Sepideh E, Hamid RE, Farin RF, Fanak F. Assessment of drug dose adjustment in patients with kidney disease: Opportunities for pharmacist involvement. Int J Pharm Pharm Sci 2012;4(3):178-81.

8. Markota NP, Markota I, Tomic M, Zelenika A. Inappropriate drug dosage adjustments in patients with renal impairment. J Nephrol 2009;22(4):497-501

9. Alahdal AM, Elberry AA. Evaluation of applying drug dose adjustment by physicians in patients with renal impairment. Saudi Pharm J 2012;20(3):217-20.

10. Henok G, Yewondwossen T, Workineh S. Drug dosage adjustment in hospitalized patients with renal impairment at Tikur Anbessa Specialized Hospital, Addis Ababa, Ethiopia. BMC Nephrol 2015;16(158):1-9.

11. Kidu G, Naod GS, Fantaahun M, Solomon A, Abrham W, Admassu A, et al. Drug dose adjustment practices in patients with renal impairment at Ayder Referral Hospital, Mekelle, Northern Ethiopia. Int J Pharm Sci Rev Res 2015;30(2):153-7.

12. Sah SK, Wanakamanee U, Lerkiatbundit S, Regmi BM. Drug dosage adjustment of patients with impaired renal function at hospital discharge in a teaching hospital. J Nepal Health Res Counc 2014;12(26):54-8.

13. Erler A, Beyer M, Petersen JJ, Saal K, Rath T, Rochon J, et al. How to improve drug dosing for patients with renal impairment in primary care - A cluster-randomized controlled trial. BMC Fam Pract 2012;13(91):1-8.

14. Prajapati A, Ganguly B. Appropriateness of drug dose and frequency in patients with renal dysfunction in a tertiary care hospital: A cross-sectional study. J Pharm Bioallied Sci 2013;5(2):136-40. 